\title{
Comparison of structure and yield of multiwall carbon nanotubes produced by the CVD technique and a water assisted method
}

\author{
Malti Bansal ${ }^{\text {a,b,c,*}, ~ C . ~ L a l ~}{ }^{\text {b }}$, Ritu Srivastava ${ }^{\text {c }}$, M.N. Kamalasanan ${ }^{\text {c }}$, L.S. Tanwar ${ }^{\text {a }}$ \\ ${ }^{a}$ Department of Physics, School of Applied Sciences, Netaji Subhas Institute of Technology, Sector-3, Dwarka, New Delhi 110075, India \\ ${ }^{\mathrm{b}}$ Carbon Technology Unit, National Physical Laboratory (Council of Scientific and Industrial Research), Dr. K. S. Krishnan Road, New Delhi 110012, India

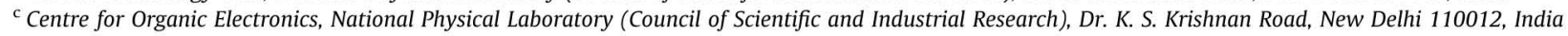

A B S T R A C T

Multiwall carbon nanotubes (MWNTs) were synthesized using chemical vapor deposition (CVD) technique and a water assisted method. Both methods produced MWNTs, which were characterized by SEM, TEM and Raman studies. It was observed that as far as quality is concerned, MWNTs produced by water assisted method are superior as the method does not employ any metal catalyst. However, as far as yield is concerned, CVD is a better method. Multiwall carbon nanotubes produced by water assisted method suffer from the drawback of low yield but have an advantage of production of multiwall carbon nanotubes without using any metal catalyst, at ambient pressure, in an environment friendly manner and using a simple and cost-effective apparatus.

\section{Introduction}

Carbon nanotubes, perhaps discovered in 1952 by Radushkevich and Lukyanovich [1,2], but glamorized in 1991 by Iijima [3], have been the centre of attention of material scientists, physicists and chemists since a long time and this has led to enormous work in the field of their synthesis [4,5], properties [6,7,8] and applications in fields as diverse as electronics $[9,10]$, photonics $[11,12]$, composites $[13,14]$, hydrogen storage $[15,16]$, etc. However, in spite of such an enormous volume of work in the field of carbon nanotubes, the scientific community is still grappling with issues related to synthesis of carbon nanotubes such as synthesis of carbon nanotubes with controlled orientations and spatial locations, synthesis of carbon nanotubes with controlled diameters and lengths, etc. As such, the field of synthesis of carbon nanotubes is still an active area of research today and novel methods of carbon nanotube synthesis are appearing in published works every other day. Some of these novel methods of carbon nanotube synthesis include synthesis of carbon nanotubes from grass [17], synthesis of carbon nanotubes by an ethanol thermal reduction process [18], one-step water assisted synthesis of high quality carbon nanotubes directly from graphite [19], synthesis of carbon nanotubes by rapid quenching of red-hot graphite rod in ethyl alcohol [20], etc. We have made an effort here to synthesize carbon nanotubes using two methods namely chemical vapor deposition (CVD) method and a water assisted method. The CVD method, though a popular technique for production of multiwall carbon nanotubes, suffers from the drawback of production of multiwall carbon nanotubes with embedded metal catalysts (used for the production of MWNTs). The implications of using metal catalysts in the production of carbon nanotubes are quite significant. The metal catalysts, which are used for the production of nanotubes such as iron, gold, cobalt, etc. can be toxic and can cause problems in clean room environments [21]. Moreover, the use of metals in nanotube synthesis makes it difficult to view the carbon nanotube formation process using the infrared spectroscopy, a challenge that has kept researchers in the dark about some of the aspects of nanotube growth [21]. Also, the metal catalysts, which are used to grow nanotubes react unfavourably with materials found in electronic circuits and composites [21,22], thus hampering the use of carbon nanotubes in these promising applications. Owing to the chemical, redox, and magnetic properties of the metal nanoparticles, interference with the corresponding tube properties cannot be avoided [23]. Thus, MWNTS produced by the CVD method are subjected to elaborate purification treatments before they can be used for applications. Removal of metal catalyst after the synthesis of carbon nanotubes requires treatment with different acids, bases, etc. The strong acid treatment, although removes the metal catalyst, but damages the surface of nanotubes, thus leading to deterioration of their electronic properties, surface properties, etc. It is difficult, if not impossible, to remove the metal catalyst particles completely after the tube production process [23]. The metal catalyst residues are incompatible with 
silicon semiconductor technology [24], thus hampering the use of carbon nanotubes in electronics. In many cases, the catalyst particles are covered by a carbon shell, which imposes additional problems for the non-destructive purification of carbon nanotubes such as by treatment with non-oxidizing acids, etc. [23]. The water assisted method for production of MWNTs reported by Enbo Wang's group [19] and later on explored by the authors $[25,26]$ has an inherent advantage of producing MWNTs without the aid of any metal catalyst and at ambient pressure. The MWNTs produced by this method do not require any further purification (for removal of metal catalyst). We present here the results obtained by us on synthesizing MWNTs by the two methods, and compare them in terms of quality and quantity of MWNTs, as evidenced by the SEM, the TEM and the Raman studies. It was observed that as far as quality is concerned, MWNTs produced by water assisted method are superior as the method does not employ any metal catalyst. However, as far as yield is concerned, CVD is a better method. Multiwall carbon nanotubes produced by water assisted method suffer from the drawback of low yield but have an advantage of producing multiwall carbon nanotubes without using any metal catalyst, at ambient pressure, in an environment friendly manner and using a simple and costeffective apparatus.

\section{Experimental details}

\subsection{Synthesis of MWNTs using the CVD method and subsequent} purification

A solution of ferrocene in toluene (in an appropriate ratio) is injected into a two stage furnace whose reaction zone is maintained at $750{ }^{\circ} \mathrm{C}$. Argon is used as the carrier gas and its flow rate is adjusted in such a manner that the maximum quantity of precursor is consumed in the reaction zone. The first stage of the furnace is maintained at around $200^{\circ} \mathrm{C}$ to ensure that the solution is vaporized as soon as it is injected (the vaporization temperature of ferrocene and toluene are 175 and $110{ }^{\circ} \mathrm{C}$, respectively). Multiwall carbon nanotubes grow predominantly in the centre of the reaction tube, which are then scraped out after cooling the reaction tube to room temperature.

The purification of as produced MWNTs was done using air oxidation (at a temperature of $350^{\circ} \mathrm{C}$ ) followed by acid treatment. A mixture of concentrated nitric and sulfuric acids in a molar ratio of $1: 3$, respectively was taken [27]. MWNTs were added to the acid mixture in an appropriate ratio and the mixture was refluxed for $20 \mathrm{~min}$. On cooling, the mixture was washed with distilled water until the mixture did not exhibit any acidity. The resultant mixture was dried at $60^{\circ} \mathrm{C}$ for further use.

\subsection{Synthesis of MWNTs using the water assisted method}

The multiwall carbon nanotubes were synthesized using the water assisted method, as reported in [19,25-26]. Briefly the method is as follows: synthetic graphite rod (99.9\% pure) was heated to red hot (above $800^{\circ} \mathrm{C}$ ) in air and then dipped into cold water $\left(\right.$ at $0^{\circ} \mathrm{C}$ ) immediately. After a slight explosion, the water turned a little turbid. This procedure was repeated many times. Finally, the solution containing formed nanomaterials was filtered using the Whatman filter paper and the products obtained were dried in oven at $60^{\circ} \mathrm{C}$ for $24 \mathrm{~h}$. No further purification treatment was carried out on the resulting nanomaterials.

\subsection{Characterization}

The formed nanomaterials were examined using scanning electron microscope (LEO SEM 440) and high resolution transmission electron microscope (Tecnai G2F-30 STWIN $300 \mathrm{kV}$ FEG). Raman spectra were recorded using Ar ion laser with an excitation wavelength of $514.5 \mathrm{~nm}$ and analyzed using a Renishaw Raman spectrometer (Invia reflex) equipped with a charge-coupled device in a back scattering geometry.

\section{Results and discussion}

\subsection{SEM studies}

Figs. 1-4 show the SEM micrographs for the raw MWNTs produced by CVD method, air oxidized MWNTs produced by the CVD method, air oxidized and acid treated MWNTs produced by the CVD method and MWNTs produced by water assisted method.

(i) Comparison of purity between the two methods: the SEM micrograph for raw MWNTs produced by the CVD method shows the presence of carbon nanotubes with length in the range of tens of micrometer and diameter in the range of tens of

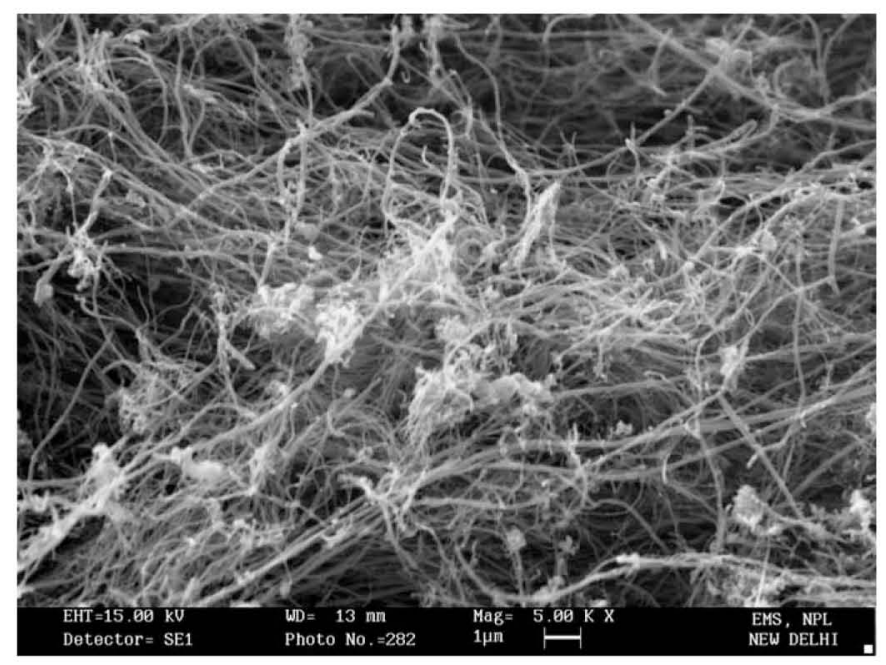

Fig. 1. Raw MWNTs synthesized by the CVD method.

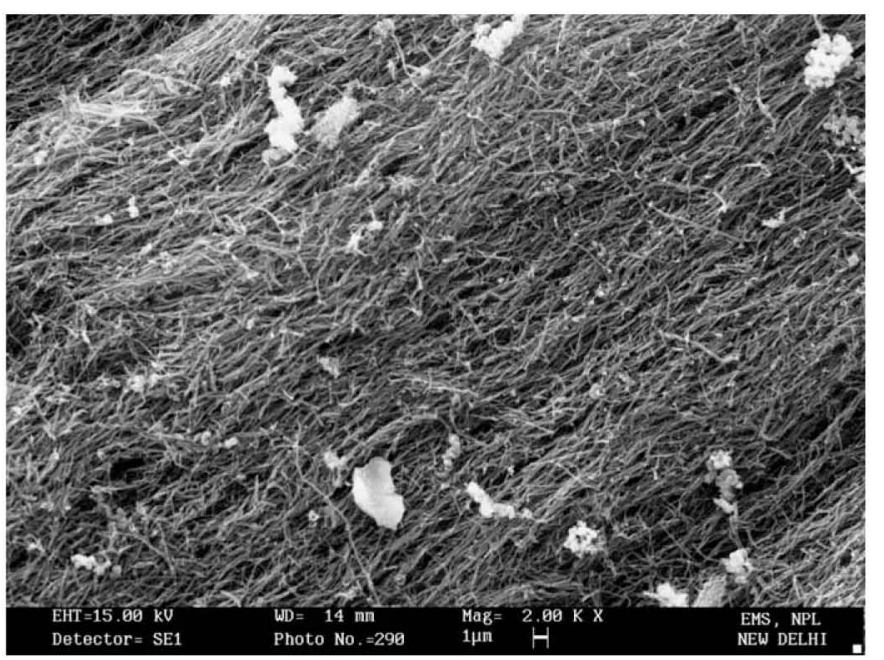

Fig. 2. Air oxidized MWNTs, which were synthesized by the CVD method. 


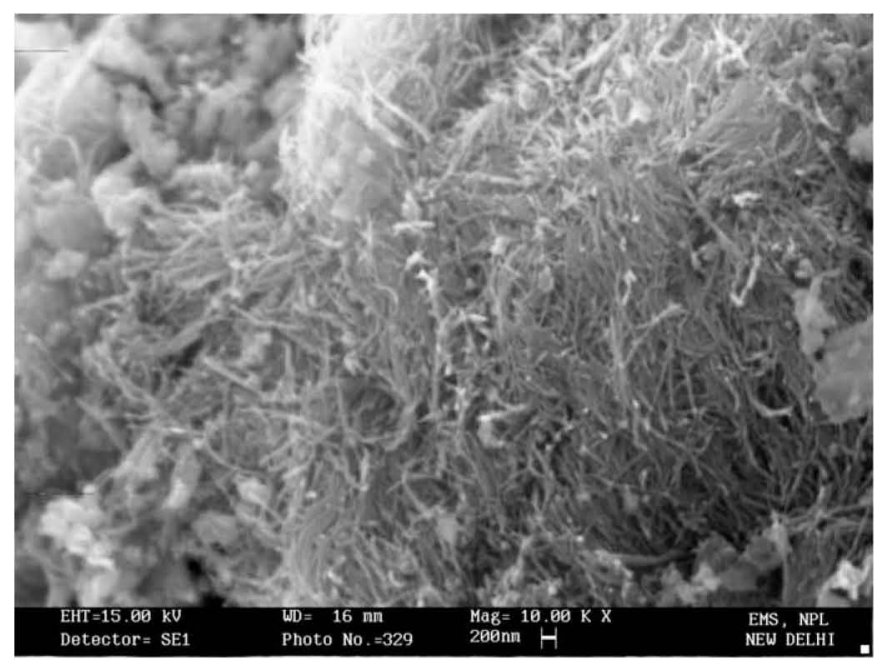

Fig. 3. Air oxidized and acid modified MWNTs, which were synthesized by the CVD method.

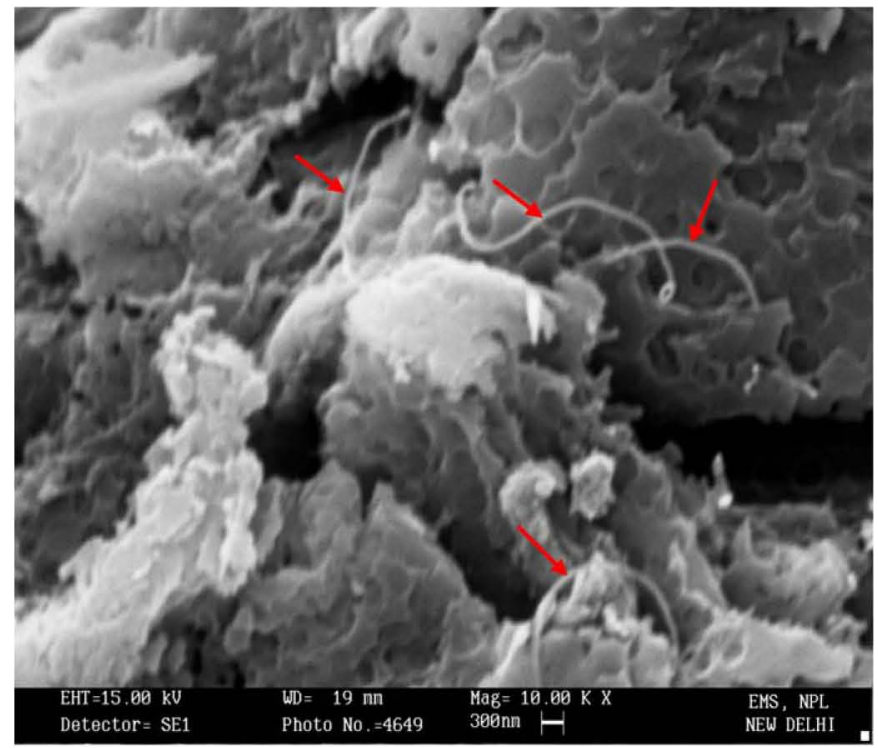

Fig. 4. MWNTs synthesized by the water assisted method. (The arrows indicate multiwall carbon nanotubes in the sample)

nanometer. The nanotubes are quite numerous in amount but contain traces of catalytic particles and large amount of amorphous carbon. The SEM micrograph for raw carbon nanotubes produced by the CVD method and subsequently subjected to air oxidation (Fig. 2) shows decrease in the amount of amorphous carbon as well as reduction in the length of carbon nanotubes. The SEM micrograph for raw carbon nanotubes produced by the CVD method and subsequently subjected to air oxidation and acid treatment (Fig. 3) shows a significant decrease in the amount of amorphous carbon and considerable shortening of the length of carbon nanotubes. However, amorphous carbon is not eliminated completely and traces of amorphous carbon are still visible. The SEM micrograph for carbon nanotubes produced by the water assisted method (Fig. 4) shows the presence of carbon nanotubes, though few in number, but free from catalyst particles (metal catalyst has not been employed in this process) and amorphous carbon. Few carbon nanotubes and exfoliated structure of initial carbon precursor (graphite) is visible.

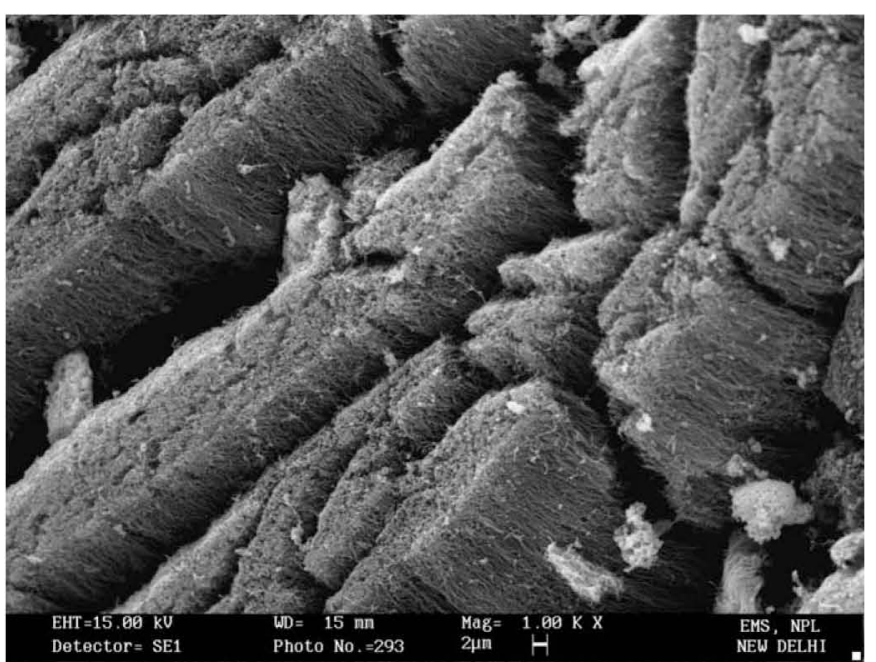

Fig. 5. Forests of Raw MWNTs synthesized by the CVD method.

(ii) Comparison of yield between the two methods: Fig. 5 shows the SEM micrograph for MWNTs synthesized by the CVD method. Dense and aligned forests of carbon nanotubes are visible. A quick look at Figs. 1-5 reveals that the numbers game is, for sure, in favour of the CVD method. The yield of carbon nanotubes by the CVD method (as visible from the SEM micrographs) exceeds the yield of carbon nanotubes by water assisted method many times.

\subsection{TEM studies}

The TEM micrograph for MWNTs synthesized by water assisted method (Fig. 6) shows the presence of nanotubes with length in the range of hundreds of nanometer, inner diameter less than $10 \mathrm{~nm}$ and outer diameter in the range of tens of nanometer $(30-50 \mathrm{~nm})$. The TEM micrograph for MWNTs synthesized by the CVD method and subsequently subjected to air oxidation and acid treatment (Fig. 7) shows the presence of nanotubes with length in the range of tens of nanometer $(50-70 \mathrm{~nm})$, inner diameter less than $10 \mathrm{~nm}$ and outer diameter in the range of tens of nanometer ( $40-60 \mathrm{~nm}$ ). Thus, nanotubes synthesized by the CVD method are shortened after being subjected to air oxidation and acid treatment whereas nanotubes synthesized by water assisted method are comparatively longer, in their pristine form. It may be pointed out that length of nanotubes can influence the nature of charge transport through carbon nanotube networks [28]. The yield of nanotubes is more using the CVD method; this point is illustrated by the TEM micrographs as well. (Figs. 6 and 7)

\subsection{Raman studies}

Fig. 8(a) shows the Raman spectra for MWNTs synthesized by water assisted method and Fig. 8(b) shows the Raman spectra for MWNTs synthesized by the CVD method. In Fig. 8(a), the sharp peak at $1581.23 \mathrm{~cm}^{-1}$ ( $\mathrm{G}$ band) corresponds to the high frequency $E_{2 \mathrm{~g}}$ first-order mode. The peak at $1354.04 \mathrm{~cm}^{-1}$ (D band) corresponds to the disorder induced mode. In Fig. 8(b), the peak at $1580 \mathrm{~cm}^{-1}$ ( $G$ band) corresponds to the high frequency $E_{2 \mathrm{~g}}$ first-order mode. The peak at $1352 \mathrm{~cm}^{-1}$ (D band) corresponds to the disorder induced mode. From the Raman spectra, we can see that the $I_{\mathrm{D}} / I_{\mathrm{G}}$ ratio for MWNTs produced by the CVD method is higher than the $I_{\mathrm{D}} / I_{\mathrm{G}}$ ratio for MWNTs produced by the water 

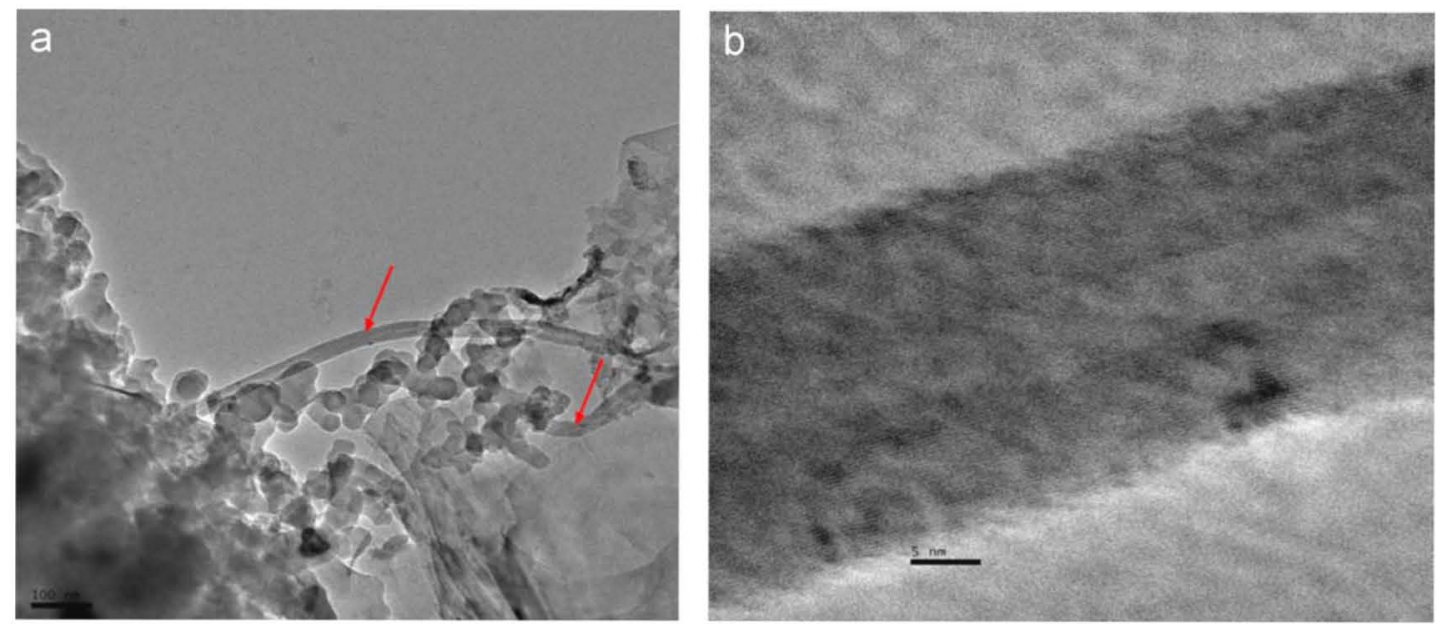

Fig. 6. The HRTEM micrographs for MWNTs synthesized by water assisted method (The arrows indicate multiwall carbon nanotubes in the sample).
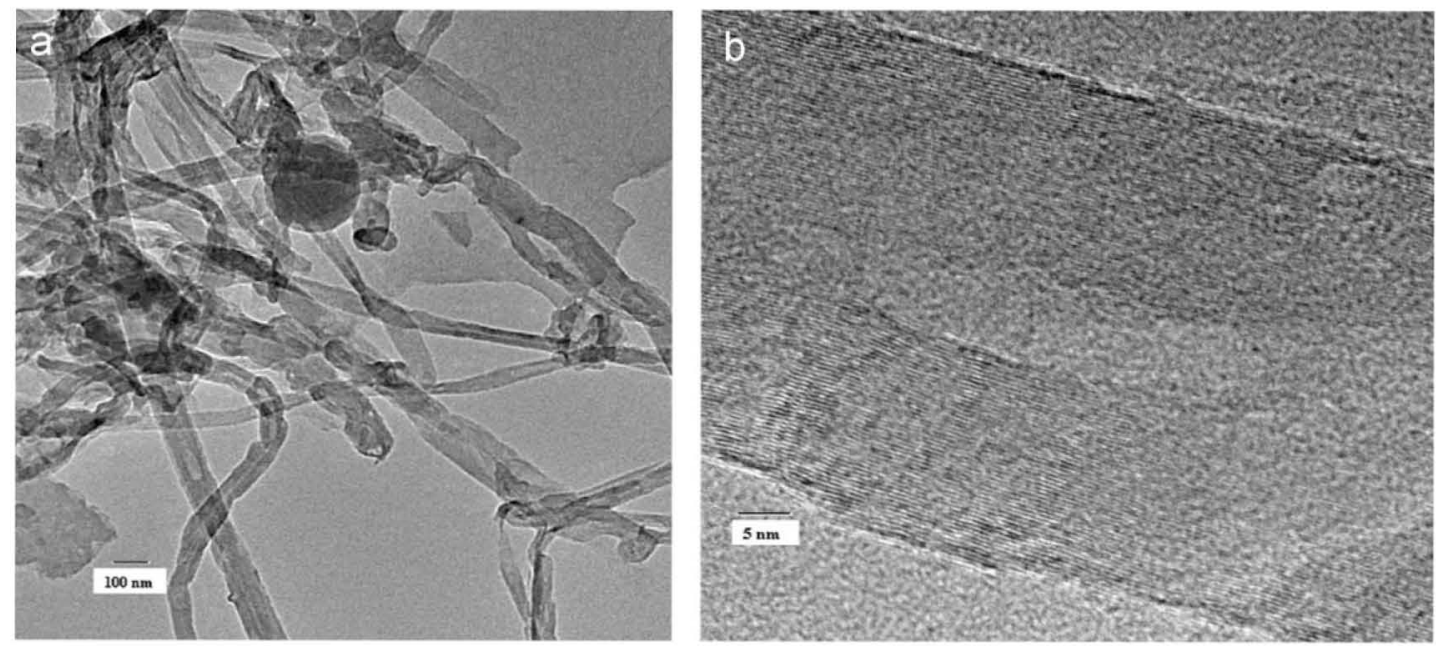

Fig. 7. The HRTEM micrographs for MWNTs synthesized by the CVD method and subsequently subjected to air oxidation and acid treatment.

assisted method. A high value of $I_{\mathrm{D}} / I_{\mathrm{G}}$ ratio indicates more number of defects and more amount of amorphous carbon in the sample $[29,30]$. Infact, the intensity of $\mathrm{D}$ band is considered defect dependent [30]. Hence, MWNTs produced by the CVD method contain more defects as compared to MWNTs produced by the water assisted method. This can be explained as follows: in the water assisted method, the basic mechanism is that when the graphite rod, at a high temperature (above $800^{\circ} \mathrm{C}$ ), is suddenly plunged into water at $0^{\circ} \mathrm{C}$, a temperature gradient comes into picture. The outer layers of graphite experience a low temperature whereas the inner core of graphite is still at a very high temperature. This produces a force that causes the outer graphite layers to dissociate from the parent graphite body and come into the surrounding water. Here, water aids in the formation of closed carbon nanotubes. Many studies [31,32] have suggested that water plays a pivotal role in the formation of closed carbon nanostructures. Thus, multiwall carbon nanotubes are formed in water in comparatively pure form (without the aid of any metal catalyst). Since MWNTs are forming directly from graphite sheets, they exhibit a higher degree of structural order, less defects and more crystalline nature, as compared to MWNTs formed by the CVD method. The MWNTs synthesized by the CVD method have low crystallinity and are covered by a thick layer of amorphous carbon, which is difficult to remove [31].

\section{Conclusions}

Multiwall carbon nanotubes were synthesized using the CVD technique and a water assisted method. It was observed that the CVD method produces multiwall carbon nanotubes in bountiful amounts; however, the raw nanotubes obtained as such are contaminated with metal catalyst particles and huge amounts of amorphous carbon. Subjecting the raw nanotubes to air oxidation and acid treatment reduces these impurities significantly but not completely. Certain applications, particularly in the field of electronics, require nanotubes in pure form, for better results. Thus, multiwall carbon nanotubes produced by the CVD method suffer from the drawback of the presence of carbonaceous and metallic impurities but have an advantage of producing multiwall carbon nanotubes in large amounts. The multiwall carbon nanotubes obtained using the water assisted method do not contain any metallic impurities as the method does not employ any metal catalyst. Moreover, carbonaceous impurities (like amorphous carbon) are also absent. The Raman spectra reveal less defects in MWNTs synthesized using the water assisted method as compared to the CVD method. Thus, multiwall carbon nanotubes produced by water assisted method suffer from the drawback of low yield but have an advantage of producing multiwall carbon nanotubes without using any metal catalyst, at 


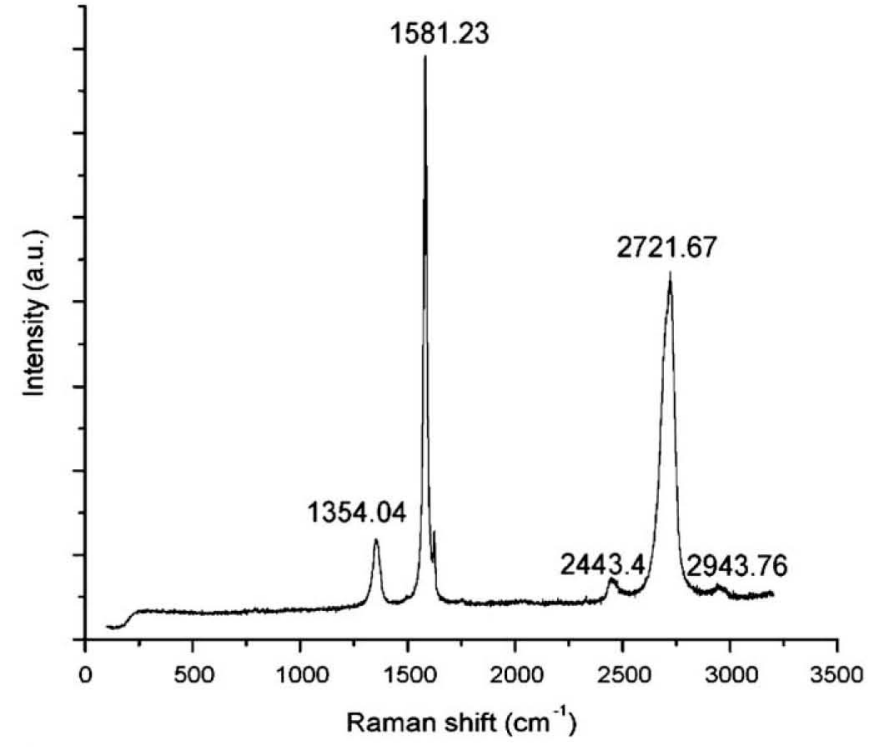

b

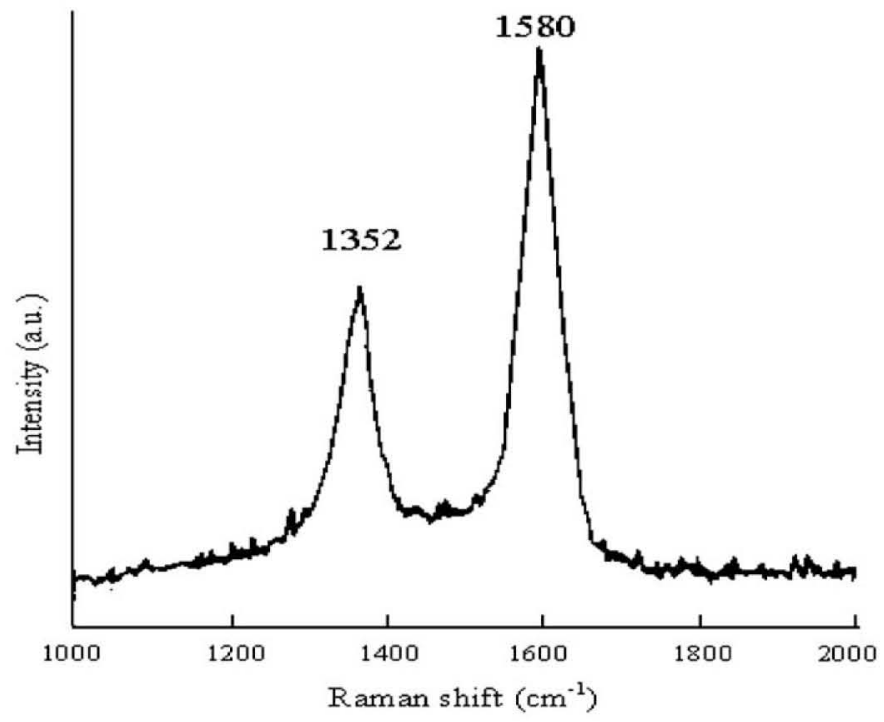

Fig. 8. (a) The Raman spectra for MWNTs synthesized by the water assisted method and (b) the Raman spectra for MWNTs synthesized by the CVD method.

ambient pressure, in an environment friendly manner and using a simple and cost-effective apparatus. Further work is required in order to increase the yield of multiwall carbon nanotubes using this novel and beneficial technique. Since multiwall carbon nanotubes are produced using this method in relatively pure form, incase further efforts lead to a boost in the yield of multiwall carbon nanotubes using this method; the multiwall carbon nanotubes so obtained can be directly employed in electronic applications like thin film transistors, field effect transistors, organic light emitting diodes, organic solar cells, organic photovoltaics, sensors, etc. where purity of nanotubes is a crucial parameter. Some efforts in this direction have been already reported [25].

\section{Acknowledgement}

One of the authors (M.B.) acknowledges the fellowship support (Teaching-cum-Research Fellowship) from Netaji Subhas Institute of Technology, New Delhi, India for carrying out this work.

\section{References}

[1] L.V. Radushkevich, V.M.O. Lukyanovich, Z. Fis. Chim. 26 (1952) 88.

[2] Marc Monthioux, Vladimir L. Kuznetsov, Carbon 44 (2006) 1621.

[3] S. Iijima, Nature 354 (1991) 56.

[4] T.W. Ebbesen, P.M. Ajayan, Nature 358 (1992) 220.

[5] N.R. Franklin, Y. Li, R.J. Chen, A. Javey, H. Dai, App. Phys. Lett. 79 (2001) 4571

[6] R. Saito, G. Dresselhaus, M.S. Dresselhaus, Physical Properties of Carbon Nanotubes, Imperial College Press, London, 1998.

[7] S. Reich, C. Thomsen, J. Maultzsch, Carbon Nanotubes, Wiley VCH, Weinheim, 2004.

[8] S.M. Bachilo, M.S. Strano, C. Kittrell, R.H. Hauge, R.E. Smalley, R.B. Weisman, Science 298 (2002) 236.

[9] R. Malti Bansal, C. Srivastava, M.N. Lal, L.S. Kamalasanan, Tanwar, Nanoscale 1 (2009) 317.

[10] J. Kong, N. Franklin, C. Zhou, M. Chapline, S. Peng, K. Cho, H. Dai, Science 287 (2000) 622.

[11] K. Kempa, B. Kimball, J. Rybczynski, Z.P. Huang, P.F. Wu, D. Steeves, M. Sennett, M. Giersig, D.V.G.L.N. Rao, D.L. Carnahan, D.Z. Wang, J.Y. Lao, W.Z. Li, Z.F. Ren, Nanoletters 3 (2003) 13.

[12] Jijun Zhao, Xiaoshuang Chen, John R.H. Xie, Anal. Chim. Acta 568 (2006) 161.

[13] P.J.F. Harris, Int. Mater. Rev. 49 (2004) 31.

[14] R. Malti Bansal, C. Srivastava, M.N. Lal, L.S. Kamalasanan, Tanwar, J. Exp. Nanosci. (2010,?) in press.

[15] M. Becher, M. Haluska, M. Hirscher, A. Quintel, V. Skakalova, U. DettlaffWeglikovska, X. Chen, M. Hulman, Y. Choi, S. Roth, V. Meregalli, M. Parrinello, R. Ströbel, L. Jörissen, M. Kappes, J. Fink, A. Züttel, I. Stepanek, P. Bernier, C. R. Phys. 4 (2003) 1055.

[16] M. Hirscher, M. Becher, J. Nanosci. Nanotechnol. 3 (2003) 3.

[17] Zhenhui Kang, Enbo Wang, Baodong Mao, Zhongmin Su, Lei Chen, Lin Xu, Nanotechnology 16 (2005) 1192.

[18] Jianwei Liu, Mingwang Shao, Xiangying Chen, Weichao Yu, Xianming Liu, Yitai Qian, J. Am. Chem. Soc. 125 (2003) 8088.

[19] Zhenhui Kang, Enbo Wang, Lei Gao, Suoyuan Lian, Min Jiang, Changwen Hu, Lin Xu, J. Am. Chem. Soc. 125 (2003) 13652.

[20] X.P. Zou, H. Abe, T. Shimizu, A. Ando, H. Tokumoto, S.M. Zhu, H.S. Zhou, Solid State Phenom 93 (2007) 121.

[21] Kate Greene, Researchers make carbon nanotubes without metal catalyst, MIT News, August 10, 2009, http://web.mit.edu/newsoffice/2009/nano tubes-0810.html.

[22] H. Liu, D. Takagi, S. Chiashi, Y. Homma, Carbon 48 (2010) 114.

[23] A. Hirsch, Angew. Chem. Int. Ed. 48 (2009) 5403.

[24] Sheng Xu, Yaguang Wei, Melanie Kirkham, Jin Liu, Wenjie Mai, Dragomir Davidovic, Robert L. Snyder, Zhong Lin Wang, J. Am. Chem. Soc. 130 (2008) 14958.

[25] Malti Bansal, C. Lal, L.S. Tanwar, V. Gupta, Mater. Sci. Eng. B 157 (2009) 93.

[26] Malti Bansal, C. Lal, R. Srivastava, M.N. Kamalasanan, L.S. Tanwar, Fullerenes, Nanotubes and Carbon Nanostructures, 2009, in press.

[27] K. Esumi, A. Ishigami, A. Nakajima, K. Sawada, H. Honda, Carbon 34 (1996) 279.

[28] Q. Cao, J.A. Rogers, Adv. Mater. 21 (2009) 29.

[29] S.P. Chai, S.H.S. Zein, A.R. Mohamed, Mater. Lett. 61 (2007) 3519.

[30] E.F. Antunes, A.O. Lobo, E.J. Corat, V.J. Trava-Airoldi, A.A. Martin, C. Verissimo, Carbon 44 (2006) 2202.

[31] J.M.C. Moreno, M. Yoshimura, J. Am. Chem. Soc. 23 (2001) 741.

[32] N. Sano, H. Wang, M. Chhowalla, I. Alexandrou, G.A.J. Amaratunga, Nature 414 (2001) 506. 